\title{
Trajectory Sensitivity Method and Master-Slave Synchronization to Estimate Parameters of Nonlinear Systems
}

\author{
Elmer P. T. Cari, Edson A. R. Theodoro, Ana P. Mijolaro, \\ Newton G. Bretas, and Luis F. C. Alberto \\ Departamento de Engenharia Elétrica da Escola de Engenharia de São Carlos, \\ Universidade de São Paulo 13566-590, São Carlos, SP, Brazil \\ Correspondence should be addressed to Luis F. C. Alberto, lfcalberto@usp.br
}

Received 1 February 2009; Revised 21 May 2009; Accepted 12 June 2009

Recommended by Elbert E. Neher Macau

\begin{abstract}
A combination of trajectory sensitivity method and master-slave synchronization was proposed to parameter estimation of nonlinear systems. It was shown that master-slave coupling increases the robustness of the trajectory sensitivity algorithm with respect to the initial guess of parameters. Since synchronization is not a guarantee that the estimation process converges to the correct parameters, a conditional test that guarantees that the new combined methodology estimates the true values of parameters was proposed. This conditional test was successfully applied to Lorenz's and Chua's systems, and the proposed parameter estimation algorithm has shown to be very robust with respect to parameter initial guesses and measurement noise for these examples.
\end{abstract}

Copyright (C) 2009 Elmer P. T. Cari et al. This is an open access article distributed under the Creative Commons Attribution License, which permits unrestricted use, distribution, and reproduction in any medium, provided the original work is properly cited.

\section{Introduction}

Several algorithms make use of synchronization to estimate parameters of nonlinear systems based on measured data. In [1, 2], for example, a Lyapunov-based design control and synchronization were used to estimate parameters of nonlinear systems. A similar approach that combines synchronization and geometric control is shown in [3]. Other attempts, including synchronization as an auxiliary tool for parameter estimation, are found in [4-6]. In addition, parameter estimation of delay systems can be found in $[7,8]$.

The measured output of the real system and the calculated output of an auxiliary system, usually taken as the mathematical model of the real system, are compared. Based on the output mismatch, parameters of the model are updated. When the outputs synchronize, that is, the difference between the real system and the model is sufficiently small, the parameters of the auxiliary system are assumed to be close enough to the real measurements. 
However, synchronization is not sufficient to ensure the correct parameter estimation. In [9], for example, the authors follow the same techniques used in [6] and verify using examples that even when the outputs synchronize, the model parameters are far from the real system parameters.

Some studies try to guarantee the correct parameter estimation when the real system and the model outputs synchronize. In [10], for example, the authors prove, for a class of dynamical systems and using a convenient Lyapunov Control Function, that the system globally synchronizes, and synchronization implies convergence to the true parameters if some special conditions are satisfied.

In this paper, master-slave synchronization framework combined with a trajectory sensitivity-based fitting algorithm is used to estimate parameters of nonlinear systems. Trajectory sensitivity method has important characteristics and advantages when compared to other approaches, such as Lyapunov Control Function framework. Trajectory sensitivitybased parameter estimation approach has the advantage of being easily implemented for any nonlinear system while other approaches like Lyapunov-based metholodoly require the existence of a Lyapunov control function. Moreover, Lyapunov-based design may require long time intervals of measured data in order to achieve synchronization while trajectory sensitivity approach can easily deal with short time intervals of measured data [1]. Another interesting feature of trajectory sensitivity approaches is that they provide estimates of the initial conditions (a desired estimate in some problems like the determination of the initial state of population density of a living species in evolutionary studies) that cannot be obtained by the Lyapunov-based approach $[1,11]$.

In spite of these advantages, it is very difficult to guarantee synchronization and convergence to the true parameters when trajectory sensitivity analysis is used as a fitting algorithm. Usually, these assumptions are not checked, and numerical tests are used to verify the robustness of the algorithm. In general, the estimation fails due to the high relative sensitivities of the trajectories with respect to parameters and initial conditions that leads to a very small convergence region of the algorithm. In this scenario, master-slave synchronization emerges as a good auxiliary tool to increase the robustness of the parameter estimation algorithm with respect to the initial guess of parameters.

Moreover, for this combined approach, there will be proposed a conditional test to ensure convergence to the true parameter values. That is, if the parameter fitting algorithm provides synchronization in a different sense, as it will explained in Section 3.1, then the convergence to the true parameters is guaranteed. Based on the trajectory sensitivity method and master-slave synchronization, some applications to estimate parameters of synchronous generator in electric power system have been developed by the authors $[12,13]$.

The structure of this paper is as follow: Section 2 presents a general framework to estimate parameters based on measured data and synchronization. In Section 3, there will be exhibited the trajectory sensitivity method. Following that, master-slave synchronization and trajectory sensitivity method will be combined and tested in two nonlinear systems; Sections 3.1 and 3.2. Moreover, the assumptions previously proposed to guarantee convergence to the true parameters will be checked for these examples. The results will be discussed in Section 4 .

\section{General Framework to Estimate Parameters Based on Measured Data and Synchronization}

A common problem that appears in many applications is the necessity of estimating parameters of a system based on the information contained in measured data (time series). 
Figure 1 illustrates the traditional framework to estimate parameters of a general nonlinear dynamical system based on measured data. In order to accomplish the estimation, an auxiliary system, which is usually taken as the mathematical model of the real system, is employed. Some of the measurements are chosen as inputs of the auxiliary system while the others are used as outputs for comparison. Using the stored measured data of the inputs, the outputs of the auxiliary system are calculated for an initial guess of parameters. Both the measured and the auxiliary system outputs are compared. Based on the output mismatch, the parameters are updated according to some prescribed rule.

The success of the general framework of Figure 1 to estimate parameters is guaranteed if the following assumptions hold.

(A1) Synchronization between the real system and the auxiliary system outputs should imply parameters of the auxiliary system sufficiently close to parameters of the real system.

(A2) The parameter fitting algorithm provides output synchronization.

Assumption (A1) is a necessary but not enough property, that has to be satisfied to guarantee the correctness of estimates. Assumption (A2) has to be satisfied to guarantee the convergence of the algorithm.

There are many alternatives to design parameter fitting algorithms; however, in most of the practical cases, it is very difficult to prove that assumption (A2) holds. In general, the success of the parameter estimation algorithm depends on personal experience to choose a convenient auxiliary system and an efficient parameter fitting algorithm. Usually, the effectiveness of the algorithms is numerically checked by means of a large number of tests, and their problems are usually related to two main aspects.

(1) The convergence region of the parameter estimation algorithm, that is a subset of the synchronization region, can be very small; that is, if a good guess of parameters is not available, then the parameter estimation algorithm diverges (synchronization is not achieved) or converges to wrong values (assumption (A1) is not satisfied).

(2) They may not be robust with respect to the presence of noise in the measurements (synchronization is not achieved due to noise presence).

In this paper, an unilateral coupling between the real and the auxiliary systems is used to enhance the robustness of synchronization. As a result, parameters of nonlinear systems (including chaotic systems) are correctly estimated, even for bad initial guesses of parameters, in the presence of measurements with noise. The coupling is of unilateral type [14], that is, some of the outputs of the real system are used as inputs to the auxiliary system. This type of coupling is known as master-slave synchronization. In this case, the real system is the master while the auxiliary system is the slave. Figure 2 illustrates the situation.

It is important to emphasize that the authors that use control Lyapunov function approach to adjust parameters have shown conditions to guarantee the satisfaction of condition (A2) for some class of systems [14]. In our case, the adjustment is accomplished by the sensitivity trajectory method using Newton's method; therefore it is not possible to exhibit an analytical condition to guarantee the satisfaction of condition (A2). As a consequence, we pursued another goal; that is, we offered a conditional proof of condition (A2); that is, (A1) is numerically checked, and if synchronization is achieved, then (A2) is true. Although the control Lyapunov approach can provide, for some classes of nonlinear systems, the satisfaction of assumptions (A1) and (A2), the motivation to study theses conditions under 


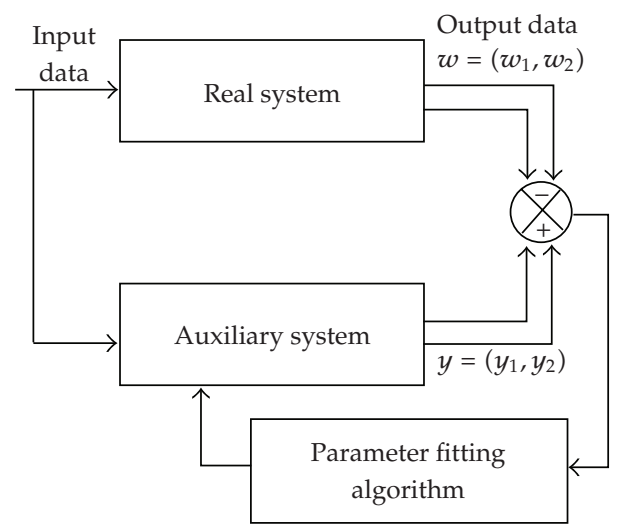

Figure 1: Framework to estimate parameters of nonlinear dynamical systems. Measured outputs of the real system are compared to the outputs of an auxiliary system. Based on the output mismatch, the fitting algorithm updates the parameters. If outputs are synchronized, parameters of the auxiliary system are assumed to be sufficiently close to parameters of the real system model.

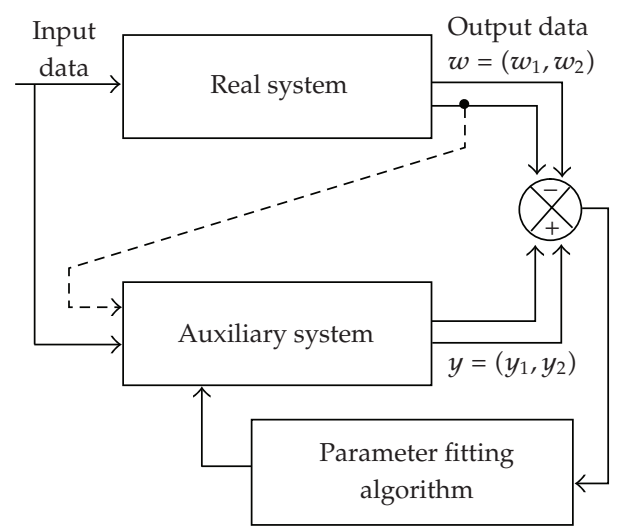

Figure 2: Parameter estimation framework with master-slave coupling between the real and the auxiliary systems. Some outputs of the real system are used as inputs of the auxiliary system.

the sensitivity trajectory method is that this method can be implemented for any nonlinear system in the form of (3.1)-(3.2), even for those we cannot exhibit a Lyapunov function.

In the next sections, this master-slave coupling approach is tested with a trajectory sensitivity-based parameter fitting algorithm.

\section{Trajectory Sensitivity Method with Master-Slave Coupling}

In this section, trajectory sensitivity analysis and master-slave synchronization will be used to estimate parameters of two chaotic systems. Although assumption (A2) cannot be easily checked, trajectory sensitivity analysis provides, from the practical point of view, advantages that justify its usage.

Trajectory sensitivity method can easily deal with hard nonlinearities. A very interesting extension of sensitivity method for differential algebraical equations (DAE systems) subject to nonsmooth events, like switchings, is presented in [15]. 
Consider a nonlinear system modeled by

$$
\begin{aligned}
\frac{d}{d t} x(t) & =f(x(t), p, u(t)), \\
y(t) & =g(x(t), p, u(t)),
\end{aligned}
$$

where $x \in R^{n}$ is the state vector, $y \in R^{m}$ is the output vector, $u \in R^{l}$ is the input vector, and $p \epsilon R^{k}$ is the parameter vector. Functions $f$ and $g$ are nonlinear, continuous, and Lipschitz with respect to $x, p$, and $u$. Let $p_{i}$ be the ith component of $p$. We assume that $f$ and $g$ are differentiable with respect to every component $p_{i}$ of $p$. In case they are not differentiable, a numerical approximation can be used to evaluate these derivatives [16]. The trajectory sensitivities $\partial x(t) / \partial p_{i}$ and $\partial y(t) / \partial p_{i}$ are computed, respectively, as

$$
\begin{aligned}
\frac{d}{d t} \frac{\partial x(t)}{\partial p_{i}} & =\frac{\partial f(x(t), p, u(t))}{\partial x} \cdot \frac{\partial x(t)}{\partial p_{i}}+\frac{\partial f(x(t), p, u(t))}{\partial p_{i}} \\
\frac{\partial y(t)}{\partial p_{i}} & =\frac{\partial g(x(t), p, u(t))}{\partial x} \cdot \frac{\partial x(t)}{\partial p_{i}}+\frac{\partial g(x(t), p, u(t))}{\partial p_{i}}
\end{aligned}
$$

Trajectory sensitivities quantify the variation of a trajectory with respect to small variations in parameters. This quantification is used to update the model parameters in order to minimize the distance between the outputs of the real system and the mathematical model.

The parameter fitting algorithm is formulated as an optimization problem; that is, we try to minimize the error function $J$, which is given by

$$
J(p)=\frac{1}{2} \int_{0}^{T_{o}}(y-w)^{t}(y-w) d t
$$

where, $w$ is the output vector of the real system, $y$ is the output of the auxiliary system (3.2), and $\left[0, T_{o}\right]$ is the time interval considered in the analysis. In other words, the minimization searches for output synchronization.

Given an initial value $p=p^{(o)}$, this optimization problem can be solved computing the sensitivity $\partial J(p) / \partial p$ and using a least squares method:

$$
G(p)=\frac{\partial J(p)}{\partial p}=\left.\int_{0}^{T_{0}} \frac{\partial y^{t}}{\partial p}(y-w) d t\right|_{p=p^{(i)}}
$$

Expanding $G(p)$ in Taylor series around $p=p^{(i)}$ and neglecting high-order terms one has

$$
G(p) \approx G\left(p^{(i)}\right)+\Gamma \Delta p
$$


where $\Gamma(p)=\partial G(p) / \partial p$. Then $\Delta p=-\Gamma^{-1} G\left(p^{(i)}\right)$. Parameters are fitted for the $i$ th iteration by $p^{(i+1)}=p^{(i)}+\Delta p^{(i+1)}$. Matrix $\Gamma(p)$ can be computed by differentiating (3.5) and neglecting higher-order terms, thus

$$
\left.\Gamma(p) \approx \int_{0}^{T} \frac{\partial y}{\partial p} \frac{\partial y}{\partial p} d t\right|_{p=p^{(i)}}
$$

When the measurements are sampled at discrete time intervals, the previous integrals are replaced by summations. For more details see reference [17].

The error function indirectly depends on the parameters. Its evaluation requires the solution of a set of ordinary differential equations (the model). Usually, nonlinear dynamic models do not have closed analytical solutions; that is, the evaluation of the error function is made via numerical integration algorithms. Newton's method, which is used to achieve synchronization, demands the evaluation of trajectory sensitivity equations that are also obtained via numerical integration algorithms. As far as we know, there is no general condition to guarantee convergence of Newton's algorithm (or similar one) in this case.

The use of trajectory sensitivities in the framework described in Figure 1 has two main problems: (i) the trajectory sensitivity method is very sensitive to initial conditions (first parameter guess), and (ii) parameters with very low sensitivities (as compared to other parameters) are not numerically identifiable due to ill-conditioned calculations. These problems become worst especially when many parameters have to be simultaneously estimated and/or chaotic behavior is present. Very often, the trajectory sensitivity method leads to erroneous estimations or even divergence of the numerical algorithm.

In this section, a master-slave coupling and trajectory sensitivity-based approach are combined to estimate parameters of chaotic systems. Comparisons between the traditional trajectory sensitivity approach (presented in Figure 1) and the proposed methodology, which includes a master-slave coupling to enhance synchronization robustness, are made in order to show the advantages of the proposed methodology.

\subsection{Lorenz's System}

Consider Lorenz's system as the real system,

$$
\begin{aligned}
& \dot{x}_{1}=-\sigma_{r} x_{1}+\sigma_{r} x_{2}, \\
& \dot{x}_{2}=-x_{2}-x_{1} x_{3}+r_{r} x_{1}, \\
& \dot{x}_{3}=-b_{r} x_{3}+x_{1} x_{2}
\end{aligned}
$$

and suppose that at least two states can be measured. Let $w=\left(x_{1}, x_{2}\right)^{T}$ be the output vector of the real system. 
In the traditional trajectory sensitivity approach, the following auxiliary system would be chosen to estimate parameters of Lorenz's system:

$$
\begin{aligned}
& \dot{z}_{1}=-\sigma z_{1}+\sigma z_{2}, \\
& \dot{z}_{2}=-z_{2}-z_{1} z_{3}+r z_{1}, \\
& \dot{z}_{3}=-b z_{3}+z_{1} z_{2} .
\end{aligned}
$$

This system has exactly the same structure as Lorenz's system model; the only difference is that the state variables $x_{i}, i=1,2,3$ were replaced by $z_{i}, i=1,2,3$, and the parameters do not have the subindex $r$ used for the real parameters. Assuming that measurements are subject to random noise, the initial conditions of states $z_{i}, i=1,2,3$ are unknown and have to be estimated. Thus, the extended parameter vector is $p_{r}=$ $\left(\sigma_{r}, r_{r}, b_{r}, z_{10}, z_{20}, z_{30}\right)$.

Our experience shows that Lorenz's system parameters cannot be simultaneously estimated using the traditional trajectory sensitivity methodology even for very small displacements of the initial conditions and parameter values from real values. Nonlinear systems with chaotic behavior, such as Lorenz's system, have a very small convergence region, because of their high relative output sensitivities with respect to the parameters.

Figure 3 shows the sensitivities of output 1 with respect to Lorenz's system parameters. The sensitivity of output $z_{1}$ with respect to parameter $\sigma$ is very small when compared with sensitivities of the $z_{1}$ output with respect to parameter $b$ or $r$. Such behavior makes the approach of Figure 1 inappropriate for parameter estimation of chaotic systems. In the particular Lorenz's system case, errors as small as $\pm 1 \%$ in the initial parameter and initial condition guesses lead the algorithm to nonconvergence due to lack of synchronization between the real system and the auxiliary system.

A master-slave coupling between the real system and the auxiliary system will be used to enhance the numerical robustness of the trajectory sensitivity-based parameter estimation algorithm. For this purpose, the next auxiliary system will be employed:

$$
\begin{aligned}
& \dot{z}_{1}=-\sigma z_{1}+\sigma z_{2}, \\
& \dot{z}_{2}=-z_{2}-x_{1} z_{3}+r x_{1}, \\
& \dot{z}_{3}=-b z_{3}+x_{1} z_{2},
\end{aligned}
$$

where $z=\left(z_{1}, z_{2}, z_{3}\right)^{T}$ is the state space vector. In this case, the states will be taken as the outputs of the auxiliary system, that is, $y=\left(z_{1}, z_{2}, z_{3}\right)^{T}$. The auxiliary system (3.10) to (3.12) resembles Lorenz's system; the difference is that state variable $z_{1}$ was replaced by the coupling variable $x_{1}$ in some convenient positions, in order to decrease nonlinearities of the auxiliary system.

The replacing of the coupling variable is accomplished in order to eliminate the nonlinear terms in the difference system (3.13). The substitution of the coupling variable $x_{1}$ in (3.10) does not have any influence in the estimate process, for being a linear term. However, we still do not have a systematic procedure to determine the best terms to substitute. 


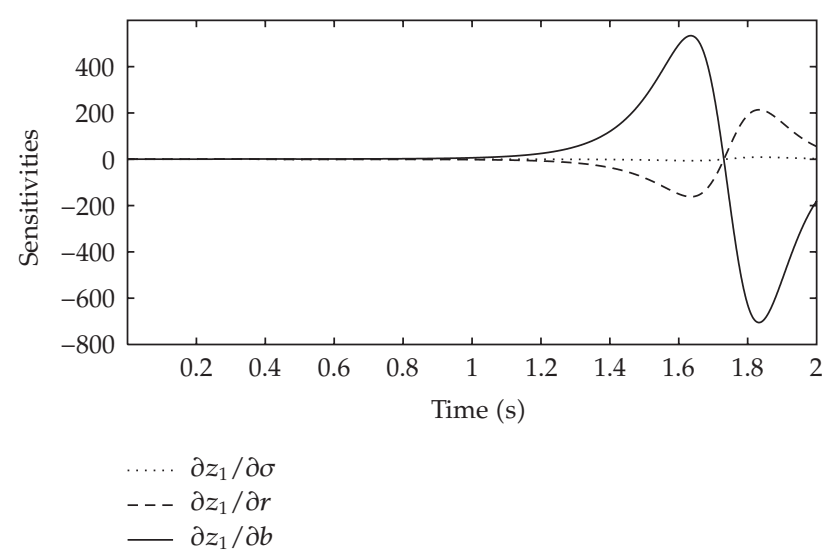

Figure 3: $Z_{1}$ output sensitivity (Lorenz's system) with respect to the parameters $\sigma, r$, and $b$.

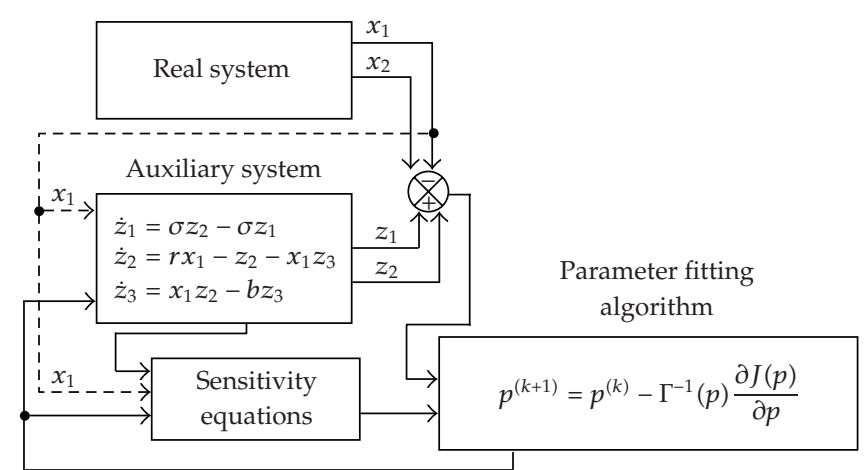

Figure 4: Master-slave trajectory sensitivity-based framework for parameter estimation of Lorenz's system parameters.

Although there is no rules in how to do this replacement, our experience shows that these modifications enlarge the stability region of the parameter estimation algorithm. Figure 4 illustrates the schematic diagram with this approach.

The error $e_{i}=z_{i}-x_{i}, i=1,2,3$ is derived from the difference between the outputs of both the auxiliary and the real systems:

$$
\begin{aligned}
& \dot{e}_{1}=-\sigma e_{1}+\sigma e_{2}+\left(x_{2}-x_{1}\right) \sigma_{e}, \\
& \dot{e}_{2}=-e_{2}-x_{1} e_{3}+x_{1} r_{e}, \\
& \dot{e}_{3}=x_{1} e_{2}-b e_{3}-x_{3} b_{e},
\end{aligned}
$$

where $\sigma_{e}=\sigma-\sigma_{r}, r_{e}=r-r_{r}$, and $b_{e}=b-b_{r}$ are the error of parameter estimation.

It is very difficult to prove assumption (A2). However, it will be shown that assumption (A1) is satisfied, for this choice of auxiliary system, in a different sense of synchronization. 
Table 1: Parameter estimation of Lorenz's system using trajectory sensitivity method including masterslave coupling with noise in the measurements.

\begin{tabular}{lccccc}
\hline Parameter & Initial value & Deviation $(\%)$ & Estimated value & True value & Error $(\%)$ \\
\hline$\sigma$ & 4.60 & $-54 \%$ & 10.034 & 10.0 & 0.34 \\
$r$ & 9.20 & $-54 \%$ & 19.96 & 20.0 & -0.2 \\
$b$ & 4.10 & $+54 \%$ & 2.66 & 2.66 & 0.00 \\
$z_{10}$ & 1.90 & $+5 \%$ & 2.07 & 2.00 & 3.5 \\
$z_{20}$ & 2.85 & $+5 \%$ & 2.94 & 3.00 & 2.0 \\
$z_{30}$ & 3.22 & $-54 \%$ & 6.96 & 7.00 & -0.57 \\
\hline
\end{tabular}

Definition 3.1. The outputs of the real system $w(t)$ and the auxiliary system $y(t) \mathcal{C}^{1}$ synchronize in the interval $\left[T_{a}, T_{b}\right]$ with precision $\varepsilon$ if

$$
\sup _{T_{a} \leq t \leq T_{b}}\|w(t)-y(t)\|+\sup _{T_{a} \leq t \leq T_{b}}\|\dot{w}(t)-\dot{y}(t)\|<\varepsilon
$$

It is possible to prove, using (3.13) and The Implicit Function Theorem, that $\mathcal{C}^{1}$ synchronization, in the sense of Definition 3.1, implies that the estimated parameters are $\varepsilon$ close to the real parameters.

Although assumption (A2) cannot be easily proven, it can be easily checked at the end of iterations. Moreover, the proposed master-slave coupling brings more robustness to synchronization. Lorenz's system parameters were estimated with good accuracy even for cases where the first guess of parameter values was displaced up to $\pm 54 \%$ from the real value. Table 1 shows the estimation results under the presence of a $\pm 5 \%$ white Gaussian random noise in all the measurements.

The outputs of the real and the auxiliary system at the beginning and at the end of iterations are shown in Figure 5.

\subsection{Chua's System}

Chua's circuit is a singular example of chaotic system, because it is the simplest circuit that exhibits this kind of phenomenon. It is composed only by one inductor, two capacitors, one resistor, and one nonlinear active resistor with a three-segment picewise-linear volt-current (V-I) characteristics, called "Chua's Diode," as shown in Figure 6.

Let the dimensionless equations of Chua's circuit [18] represent the real system:

$$
\begin{aligned}
\dot{x}_{1} & =\alpha_{r}\left(x_{2}-x_{1}-f\left(x_{1}\right)\right), \\
\dot{x}_{2} & =x_{1}-x_{2}+x_{3}, \\
\dot{x}_{3} & =-\beta_{r} x_{2}-\gamma_{r} x_{3}, \\
f\left(x_{1}\right) & =b x_{1}+\frac{1}{2}(a-b)\left\{\left|x_{1}+1\right|-\left|x_{1}-1\right|\right\},
\end{aligned}
$$

where $\alpha, \beta, \gamma, a$, and $b$ are the parameters to be estimated. We assume that states are measured; that is, $w=\left(x_{1}, x_{2}, x_{3}\right)^{T}$ is the output vector of the real system. Assuming that the 


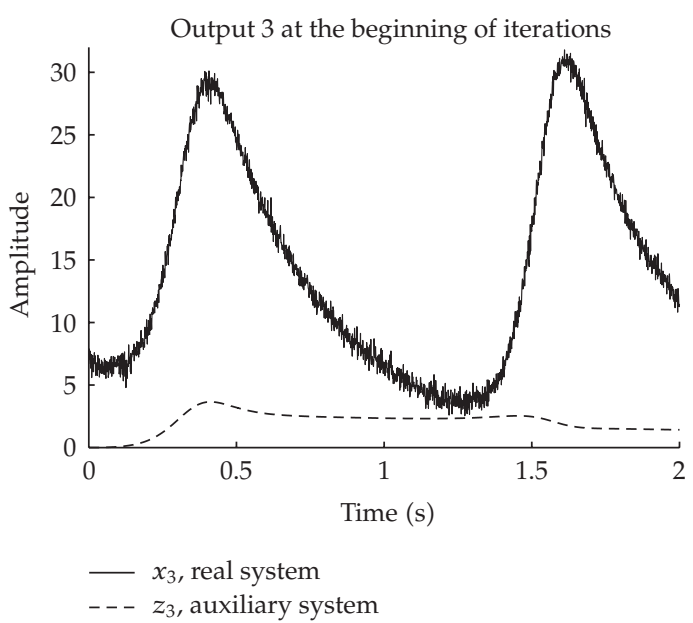

(a)

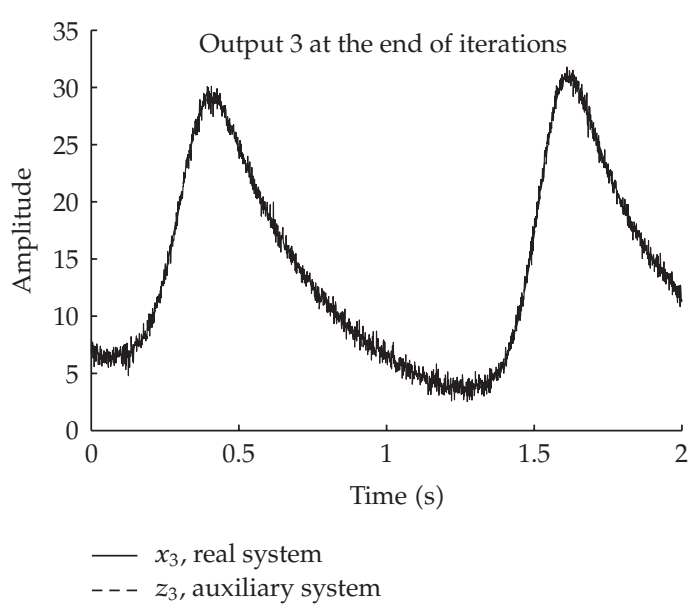

(b)

Figure 5: Comparison between the outputs of the auxiliary system at the beginning and at the end of iterations for output number 3. A white Gaussian noise with zero mean and standard deviation of $5 \%$ of the highest measured value was applied to all measurements.

initial conditions of differential equations of the model are unknown and they also have to be estimated. Thus, the extended parameter vector is $p=\left(\alpha, \beta, \gamma, a, b, x_{10}, x_{20}, x_{30}\right)$, whose true values are $\alpha_{r}=6.5792, \beta_{r}=10.9024, \gamma_{r}=-0.0445, a_{r}=-1.1829, b_{r}=-0.6524, x_{1 o}=0.15$, $x_{2 o}=0.90$, and $x_{3 o}=0.80$. Measurements of the real system were obtained by numerical integration of (3.15) to (3.18), with their true parameters and initial conditions. Random white Gaussian noise of $\pm 5 \%$ of the peak value was added to the real measurements.

Like Lorenz's system, parameters of Chua's circuit cannot be simultaneously estimated using the traditional trajectory sensitivity approach of Figure 1 due to high relative sensitivity of trajectories with respect to parameters and initial conditions. Our experience shows that errors as small as $\pm 1 \%$ on the initial parameter guesses lead the algorithm to nonconvergence due to lack of synchronization.

In order to overcome this difficulty, the following auxiliary system was chosen:

$$
\begin{aligned}
\dot{z}_{1} & =\alpha\left(z_{2}-x_{1}-f\left(x_{1}\right)\right)-k\left(z_{1}-x_{1}\right), \\
\dot{z}_{2} & =x_{1}-z_{2}+z_{3} \\
\dot{z}_{3} & =-\beta z_{2}-\gamma z_{3} \\
f\left(x_{1}\right) & =b x_{1}+\frac{1}{2}(a-b)\left\{\left|x_{1}+1\right|-\left|x_{1}-1\right|\right\} .
\end{aligned}
$$

The auxiliary system resembles Chua's circuit model; the difference is that a masterslave coupling between the real and the auxiliary systems was employed; that is, state variable $z_{1}$ was replaced by the measured real variable $x_{1}$ in some convenient positions to reduce nonlinearities in the auxiliary system. Moreover, to enhance synchronization robustness, an extra term was added to (3.15), $g\left(z_{1}, x_{1}\right)=-k\left(z_{1}-x_{1}\right)$, where $k=10$. Figure 7 illustrates this parameter estimation scheme. 


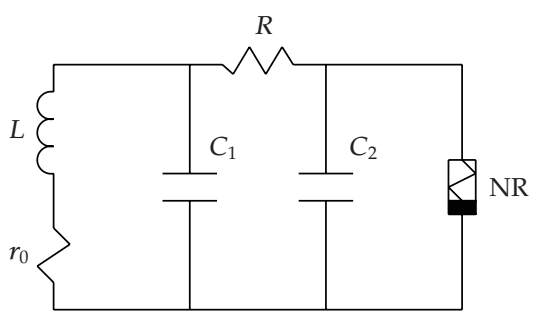

(a)

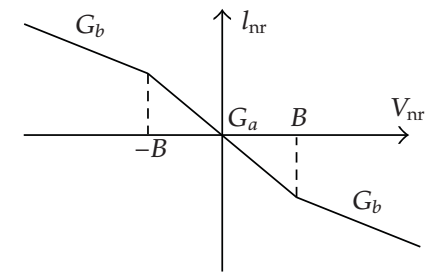

(b)

Figure 6: Chua's circuit and the three-segment odd-symmetric picewise-linear volt-current (V-I) characteristic of Chua's diode.

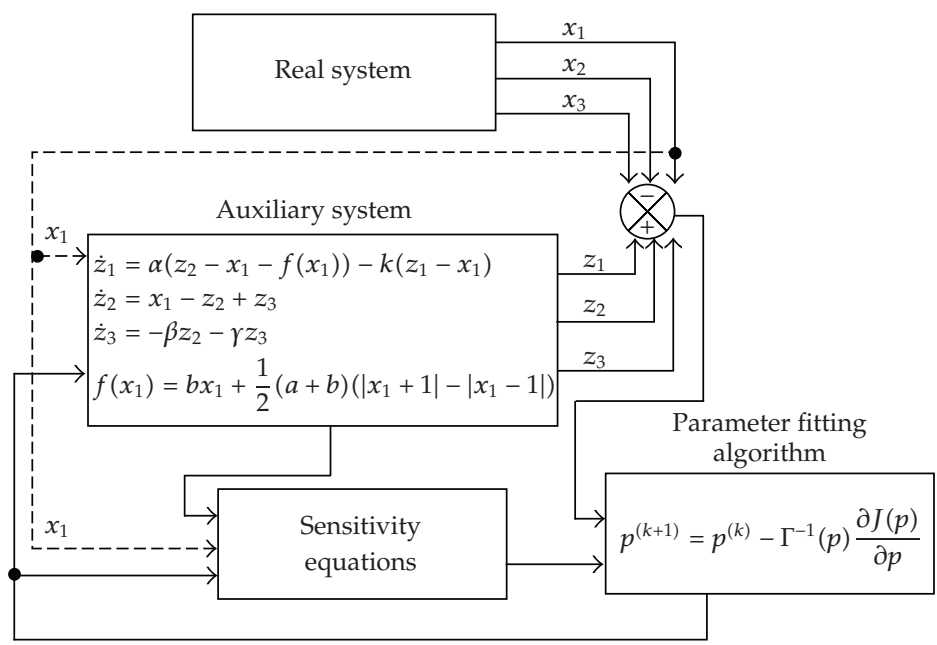

Figure 7: Master-slave trajectory sensitivity based framework for parameter estimation of Chua's circuit.

The error $e_{i}=z_{i}-x_{i}, i=1,2,3$ is derived from the difference between the outputs of both the auxiliary and the real systems:

$$
\begin{aligned}
\dot{e}_{1}= & -b e_{1}+\alpha e_{2}+\alpha_{e}\left(x_{2}-x_{1}\right)-x_{1}\left(\alpha b_{e}-b_{r} \alpha_{e}\right) \\
& -\frac{1}{2}\left[\alpha_{e}\left(a_{r}-b_{r}\right)-\alpha\left(a_{e}-b_{e}\right)\right]\left\{\left|x_{1}+1\right|-\left|x_{1}-1\right|\right\}, \\
\dot{e}_{2}= & -e_{2}+e_{3}, \\
\dot{e}_{3}= & -\beta e_{2}-\gamma e_{3}-x_{3} \gamma_{e}-x_{2} \beta_{e},
\end{aligned}
$$

where $\alpha_{e}=\alpha-\alpha_{r}, \beta_{e}=\beta-\beta_{r}, \gamma_{e}=\gamma-\gamma_{r}, a_{e}=a-a_{r}$, and $b_{e}=b-b_{r}$ are the error of parameter estimation.

It is practicable proved that $\mathcal{C}^{1}$-synchronization of outputs, in the sense of Definition 3.1, implies that parameters of the auxiliary system $p=(\alpha, \beta, \gamma, a, b)$ are $\varepsilon$-close 


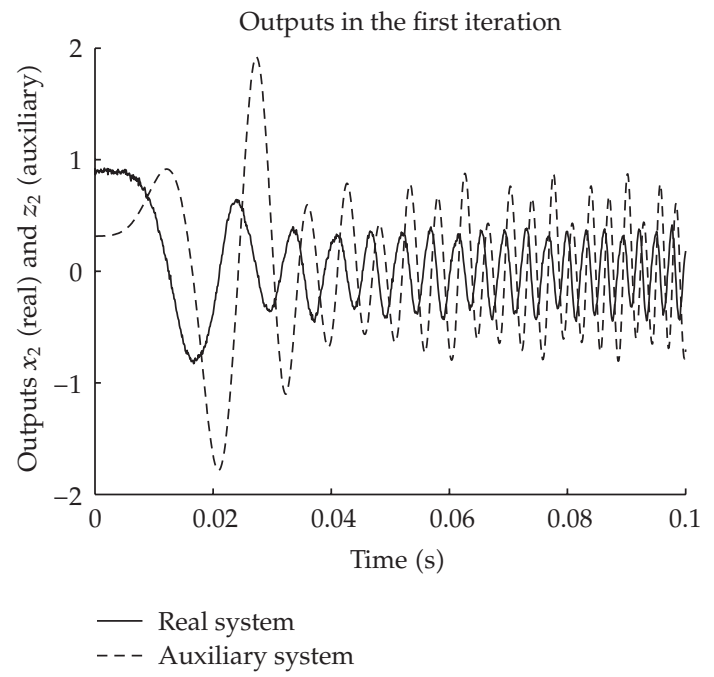

(a)

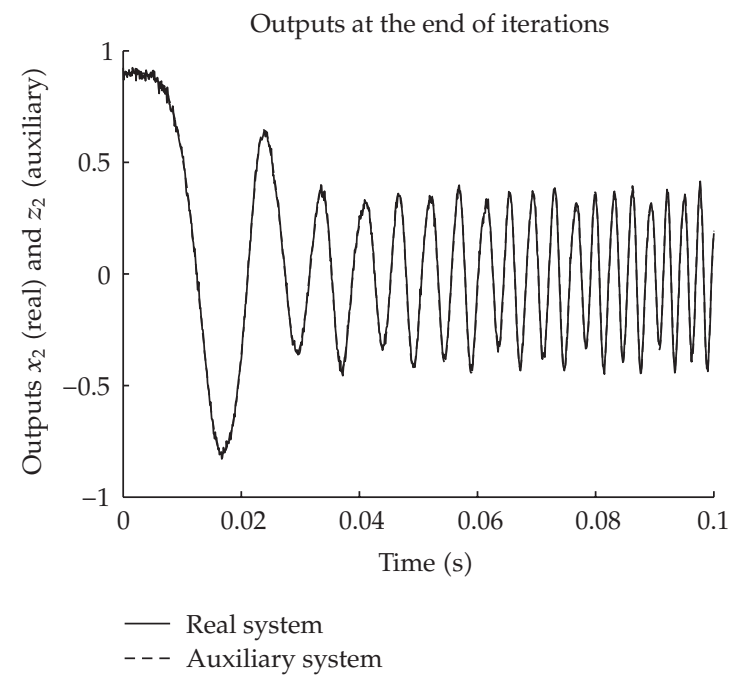

(b)

Figure 8: Comparison between outputs of real and auxiliary systems at the beginning and at the end of iterations for the output number 2. A white Gaussian noise with mean zero and standard deviation of $5 \%$ of the highest measurement value was applied to all measurement.

to the real parameter values $p_{r}=\left(\alpha_{r}, \beta_{r}, \gamma_{r}, a_{r}, b_{r}\right)$; that is, assumption (A1) is satisfied. For this purpose,the Implicit Function Theorem and (3.20) must be used.

Although assumption (A2) cannot be easily proven, it can be checked at the end of the iterations. Chua's circuit parameters were successfully estimated even for parameter initial guess deviations as large as $65 \%$ from the real values. Table 2 shows the estimation results.

The output of the real and the auxiliary system at the beginning and at the end of iterations are shown in Figure 8. 
Table 2: Parameter estimation of Chua's system using trajectory sensitivity method including master-slave coupling with noise in the measurements.

\begin{tabular}{lccccc}
\hline Parameter & Initial value & Deviation & Estimated value & True value & Error $(\%)$ \\
\hline$\alpha$ & 2.3027 & $-65 \%$ & 6.5435 & 6.5792 & 0.54 \\
$\beta$ & 3.8158 & $-65 \%$ & 10.8906 & 10.9024 & 0.11 \\
$\gamma$ & -0.0156 & $-65 \%$ & -0.0444 & -0.0445 & 0.27 \\
$a$ & -0.4137 & $-65 \%$ & -1.1807 & -1.1820 & 0.12 \\
$b$ & -0.2283 & $-65 \%$ & -0.6539 & -0.6524 & 0.24 \\
$x_{0}$ & 0.0525 & $-65 \%$ & 0.1542 & 0.1500 & 2.83 \\
$y_{0}$ & 0.3150 & $-65 \%$ & 0.9009 & 0.9000 & 0.10 \\
$z_{0}$ & 0.2800 & $-65 \%$ & 0.8185 & 0.8000 & 2.31 \\
\hline
\end{tabular}

\section{Conclusions}

A combination of trajectory sensitivity method and master-slave synchronization was proposed to parameter estimation of nonlinear systems. It was shown that master-slave coupling increases the robustness of the trajectory sensitivity algorithm with respect to the initial guess of parameters. Since synchronization is not a guarantee that the estimation process converges to the correct parameters, a conditional test that guarantees the new combined methodology estimates that the true values of parameters was proposed. This conditional test was successfully applied to Lorenz's and Chua's systems and the proposed parameter estimation algorithm has shown to be very robust with respect to parameter initial guesses and measurement noise for these examples.

\section{Acknowlegments}

This research was supported in part by Fundação de Amparo a Pesquisa do Estado de São Paulo (FAPESP) and Conselho Nacional de Desenvolvimento Cientfico e Tecnológico (CNPq).

\section{References}

[1] D. Huang and R. Guo, "Identifying parameter by identical synchronization between different systems," Chaos, vol. 14, no. 1, pp. 152-159, 2004.

[2] R. Konnur, "Synchronization-based approach for estimating all model parameters of chaotic systems," Physical Review E, vol. 67, no. 2, Article ID 027204, 2003.

[3] U.S. Freitas, E. E. N. Macau, and C. Grebogi, "Using geometric control and chaotic synchronization to estimate an unknown model parameter," Physical Review E, vol. 71, no. 4, Article ID 047203, 4 pages, 2005.

[4] Y. Crispin, "A fluid dynamical approach to the control, synchronization and parameter identification of chaotic systems," in Proceedings of the American Control Conference, vol. 3, pp. 2245-2250, May 2002.

[5] P. M. Ines and M. Joaquin, "Adaptative approximation method for joint parameter estimation and identical synchronization of chaotic systems," Physical Review E, vol. 72, Article ID 057202, 2005.

[6] U. Parlitz, "Estimating model parameters from time series by autosynchronization," Physical Review Letters, vol. 76, no. 8, pp. 1232-1235, 1996.

[7] D. Ghosh and S. Banerjee, "Adaptive scheme for synchronization-based multiparameter estimation from a single chaotic time series and its applications," Physical Review E, vol. 78, no. 5, Article ID 056211, 2008. 
[8] D. Ghosh, "Nonlinear-observer-based synchronization scheme for multiparameter estimation," Europhysics Letters, vol. 84, no. 4, Article ID 40012, 4 pages, 2008.

[9] L. Li, H. Peng, X. Wang, and Y. Yang, "Comment on two papers of chaotic synchronization," Physics Letters A, vol. 333, no. 3-4, pp. 269-270, 2004.

[10] W. Yu, G. Chen, J. Cao, J. Lü, and U. Parlitz, "Parameter identification of dynamical systems from time series," Physical Review E, vol. 75, Article ID 067201, 4 pages, 2007.

[11] M. R. Gameiro and H. M. Rodrigues, "Applications of robust synchronization to communication systems," Applicable Analysis, vol. 79, no. 1-2, pp. 21-45, 2000.

[12] E. P. T. Cari, L. F. C. Alberto, and N. G. Bretas, "A methodology for parameter estimation of synchronous generators based on trajectory sensitivity and synchronization technique," in Proceedings of IEEE Power Engineering Society General Meeting (PES '06), June 2006.

[13] E. P. T. Cari, L. F. C. Alberto, and N. G. Bretas, “Metodologia para a estimao de parmetros baseada na tcnica de sensibilidade de trajetria e conceitos de sincronizao," in Proceedings of the Anais16th Congresso Brasileiro de Automtica, October 2006.

[14] L. M. Pecora and T. L. Carroll, "Synchronization in chaotic systems," Physical Review Letters, vol. 64, no. 8, pp. 821-824, 1990.

[15] I. A. Hiskens, "Nonlinear dynamic model evaluation from disturbance measurements," IEEE Transactions on Power Systems, vol. 16, no. 4, pp. 702-710, 2001.

[16] S. M. Benchluch and J. H. Chow, "A trajectory sensitivity method for the identification of nonlinear excitation system models," IEEE Transaction on Energy Conversion, vol. 8, pp. 159-164, 1993.

[17] J. B. Cruz, Feedback System, McGraw-Hill, New York, NY, USA, 1972.

[18] L. O. Chua, C. W. Wu, A. Huang, and G. Zhong, "A universal circuit for studying and generating chaos-part I: routes to chaos," IEEE Transactionson Circuits and Systems, vol. 40, no. 10, pp. 732-744, 1993. 


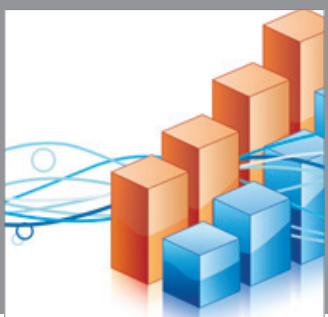

Advances in

Operations Research

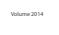

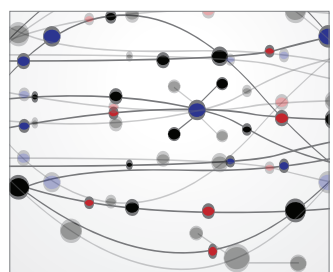

\section{The Scientific} World Journal
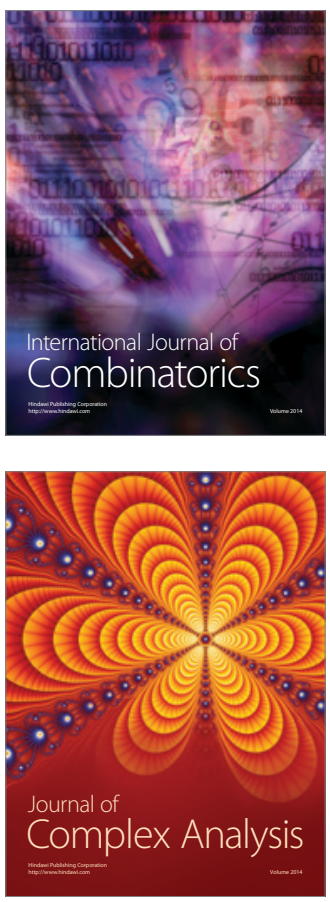

International Journal of

Mathematics and

Mathematical

Sciences
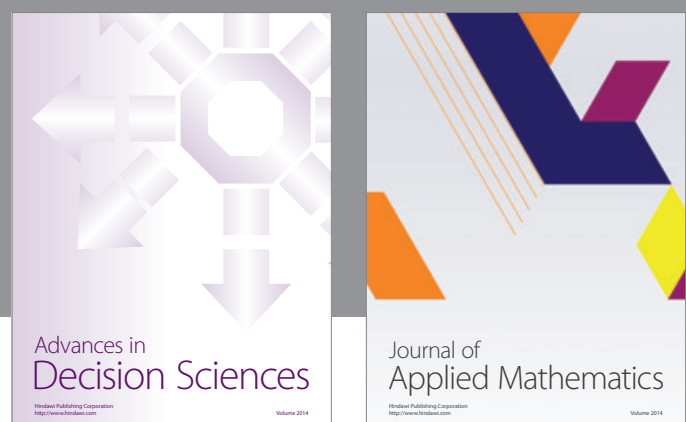

Journal of

Applied Mathematics
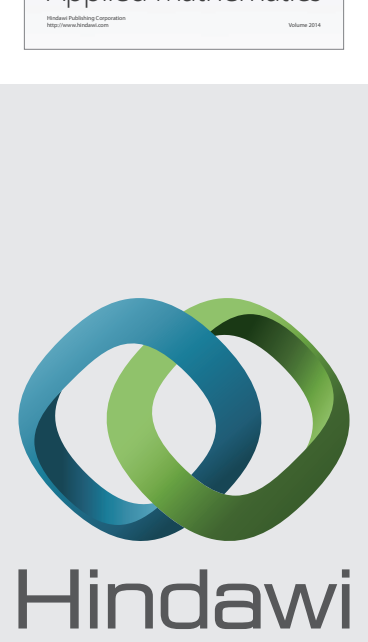

Submit your manuscripts at http://www.hindawi.com
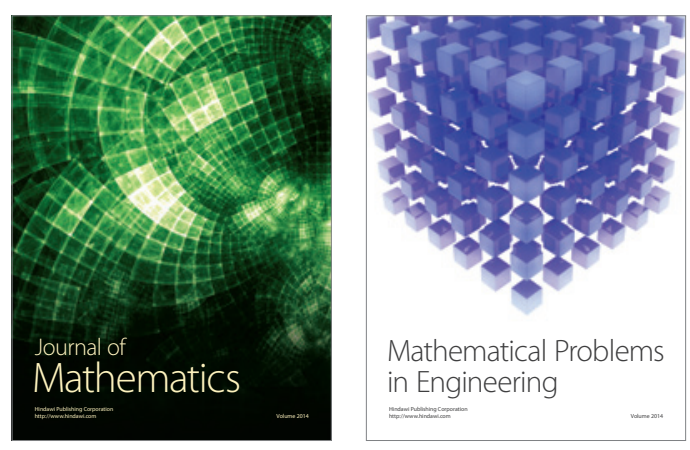

Mathematical Problems in Engineering
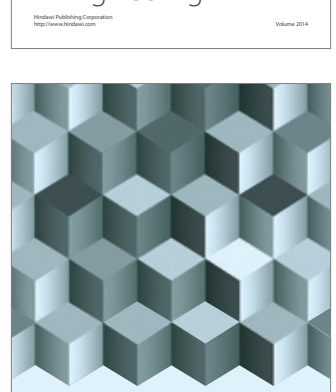

Journal of

Function Spaces
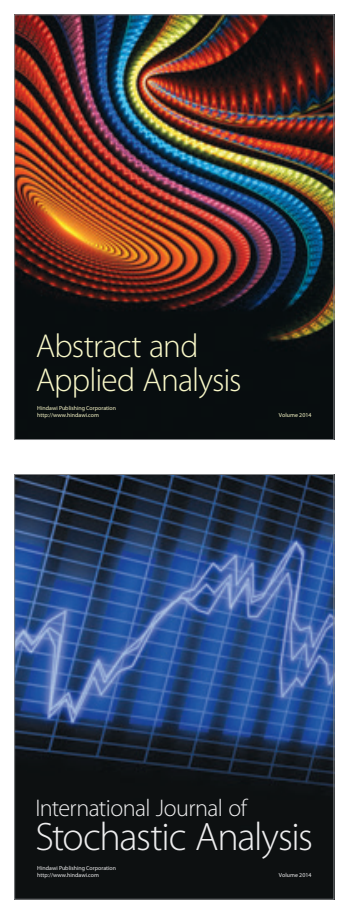

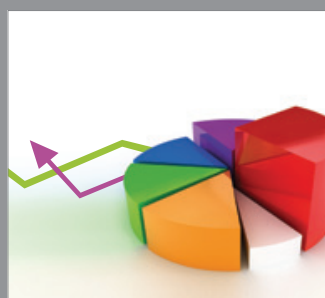

ournal of

Probability and Statistics

Promensencen
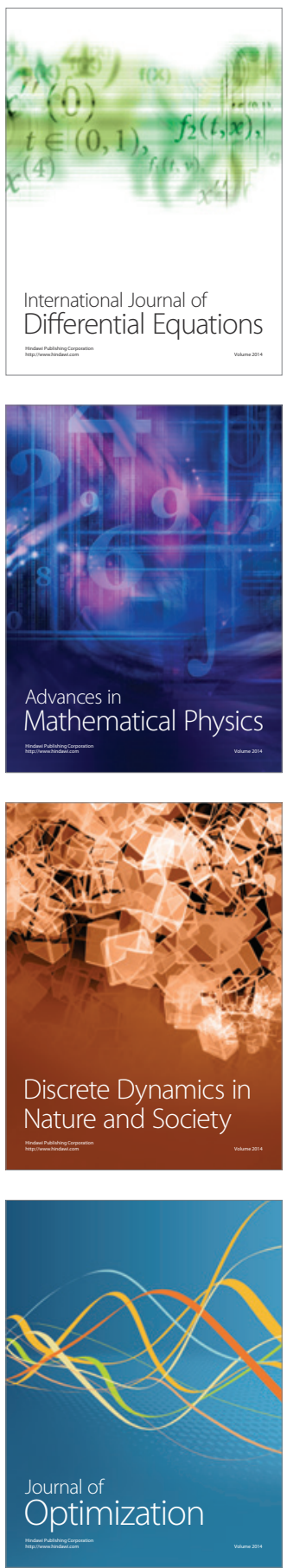